\title{
MÉTODO PARA DETERMINAR O DIÂMETRO MÁXIMO DE CORTE EM FLORESTAS TROPICAIS UTILIZANDO-SE O GRÁFICO "UPLOT-DAP".
}

\author{
Ulisses Silva da CUNHA ${ }^{1}$, Sebastião do Amaral MACHADO ${ }^{2}$, Afonso \\ FIGUEIREDO FILHO ${ }^{3}$, Roberto Tuyoshi HOSOKAWA ${ }^{4}$
}

\begin{abstract}
RESUMO - A presente pesquisa apresenta um método de determinação do diâmetro máximo de corte para florestas tropicais utilizando-se a construção gráfica denominada de "Uplot-Dap". O objetivo da pesquisa foi detectar a existência e locação de um possível diâmetro máximo em função do equilíbrio biológico das espécies em uma floresta clímax, utilizando-se o método gráfico "Uplot-Dap". Para testar o método gráfico foram utilizados 28320 DAP com casca de árvores procedentes de 15 blocos de 96 ha da Floresta Nacional do Tapajós, Belterra, Pará. Os gráficos foram construídos considerando uma amplitude de classe diamétrica de $10 \mathrm{~cm}$ adotada como valor de referência. O método "Uplot-Dap" que utiliza técnica de análise visual de dados como forma de interpretação da dispersão dos diâmetros, mostrou-se bastante flexível e essencialmente prático. Através do método foi possível fixar o $\mathrm{DAP}=95 \mathrm{~cm}$ como um indicador do diâmetro máximo de explorabilidade a ser adotado na primeira intervenção como valor de referência nas operações de corte para esse tipo de floresta. Todavia, esse ponto pode variar de floresta para floresta e sua nitidez depende do tipo de floresta e suas condições estruturais. A maior contribuição do método gráfico consistiu em respeitar as propriedades das classes diamétricas em florestas naturais que por razões biológicas ou estágio sucessional, expressem a sua distribuição diamétrica como uma função de eventos raros, nulos ou probabilísticos. A eficiência do método baseia-se na sua capacidade de controlar a variação dos dados de modo que as análises sejam mais consistentes com os fatores físicos e biológicos que caracterizam a dinâmica de florestas naturais.
\end{abstract}

Palavras-Chave: Diâmetro máximo, Método gráfico, Floresta tropical, "Uplot-Dap"

Method for Determination of the Maximum Diameter of Cut in Tropical Forests Using the Graphic "Uplot-Dap".

ABSTRACT - This research presents a method for determination of the maximum diameter of cut in tropical forests being used the denominated graphic construction of 'Uplot-Dap". The main objective consisted on detecting the existence and location of a possible maximum diameter in function of the biological balance of the species in a climax forest. To test the graphic method they were used $28320 \mathrm{DBH}(\mathrm{cm})$ outside bark coming from 15 blocks of 96 hectares is of the National Forest of Tapajós, Belterra, Pará, Brazil. The method that uses technique of visual analysis of data as a way of interpretation of the dispersion of the diameters, was shown quite flexible and essentially practical. The graphs were built considering a width of diameter class of $10 \mathrm{~cm}$ adopted as reference value. Through this method it was possible to fasten the $\mathrm{DBH}=95 \mathrm{~cm}$ as an indicator of the maximum diameter of exploitability to be adopted in the 1st intervention as reference value in the cut operations for this forest type. Mean while this point can vary from forest to forest and its clearness depends on the forest type and its structural condition. The largest contribution of the graphic method consisted on respecting the properties of the diameter classes in natural forests that for biological reasons or sucessional estate, express its diameter distribution as a function of rare, null or probabilistics events. The efficiency of the method bases on its capacity of controlling the variation of the data so that the analysis are more consistent with the physical and biological factors that characterize the dynamics of natural forests.

Key-words: Maximum diameter, Graphic solution, "Uplot-Dap", Tropical forest

${ }^{1}$ UFAM/Doutorando em Manejo Florestal pela UFPR. Av. Gal. Rodrigo Otávio J. Ramos, 3000, Aleixo, Mini-Campus, 69077-000, Manaus-AM. Fone/Fax: (0xx92) 647-4042/647-4043. E-mail: uscfua@ig.com.br/ucunha@fua.br.

${ }^{2}$ Curso de Engenharia Florestal da UFPR. PQ 1A - CNPq. E-mail: sammac@floresta.ufpr.br.

${ }^{3}$ Curso de Engenharia Florestal da UFPR. PQ 2A - CNPq. E-mail: afig@floresta.ufpr.br.

${ }^{4}$ Curso de Engenharia Florestal da UFPR. PQ 1B-CNPq. E-mail: hosokawa@floresta.ufpr.br. 


\section{Introdução}

O diametro mínimo de corte é regulamentado por legislação específica. Para as florestas da Amazônia Legal, a portaria $\mathrm{N}^{\circ} 048 \mathrm{de}$ 17 de julho de 1995 do Instituto Brasileiro do Meio Ambiente e dos Recursos Naturais Renováveis (IBAMA) estabeleceu que o DAP $\geq 45 \mathrm{~cm}$ deve ser o diâmetro mínimo de corte. A idéia do estabeleceimento de um diâmetro máximo de corte baseia-se na necessidade de preservação das espécies, especialmente espécies com estratégias de reprodução pouco conhecidas.

Do ponto de vista de quem explora a floresta, existe sempre uma resistência natural a esse tipo de exigência já que a retirada dos maiores indivíduos parece não ter sérias implicações, pois alguns podem estar em fase senil ou próximo de alcançarem a morte natural. Visto sob o aspecto físico e fitossanitário admite-se que indivíduos de grandes diâmetros podem ter atingido a produção assintótica e a melhor alternativa seria o seu aproveitamento integral. Mas em termos de equilíbrio é importante manter representantes das espécies nos dois extremos, pois a sucessão não pode prescindir dessas duas fontes de diversidade genética.

Em termos de manejo florestal, restringir o corte das árvores de grandes diâmetros poderia ter implicações na redução do volume comercial a ser explorado, tendo como consequência imediata comprometimento da viabilidade econômica do empreendimento. Mas considerando que esse volume tem baixo impacto sobre o volume total, incluindo as perdas e substituições nos procedimentos de manejo, é medida das mais acertadas manter esses indivíduos principalmente por ocasião do primeiro corte quando as consequências de todas as medidas adotadas ainda não são bem conhecidas.

Como recurso natural renovável a floresta Amazônica é única no gênero devido a sua complexidade estrutural e grande número de espécies com características e propriedades bem distintas. O uso crescente de madeiras de florestas tropicais e a exigência dos consumidores, determinaram o surgimento de uma escala de preferências. Apesar da escassez de alguns tipos de madeiras como o mogno (Swietenia macrophylla King), o mercado que é conservador continua exercendo pressão sobre poucas espécies com alto nível de demanda, enquanto outras são raramente aceitáveis. Mas no outro extremo desta escala existe um grande número de espécies que em geral denomina-se de "espécies pouco conhecidas" (EPC), "indesejáveis" ou de "exploração não necessária". Assim, poder-se-ia definir uma EPC como uma espécie pouco aceita comercialmente e que fica na florestadepois da exploração florestal.

Apesar das atuais limitações, a lista das espécies comerciais tem aumentado por causa dos avanços tecnológicos, das atividades de propa- 
ganda e da crescente escassez das espécies de maior preferência. Mesmo com um mercado interno menos exigente que a exportacão, em geral só se aproveita uma pequena proporção das espécies potencialmente utilizáveis. Ao examinar os aspectos técnicos da proporção das EPC em Papua Nova Guiné, Eddowes (1980) identificou os seguintes problemas:

- dificuldade para identificar as espécies madeireiras;

- dados insuficientes sobre as características físicas e mecânicas;

- comercialização incorreta orientada para usos finais errôneos;

- fornecimentos irregulares ou insuficientes e,

- classificação deficiente.

Nem sempre as espécies de maior interesse comercial são as de maiores diâmetros.

Existem razões suficientes para acreditar que as EPC apresentam um papel estratégico importante nas opções de manejo, na medida em que algumas apresentam área basal $\left(\mathrm{m}^{2} / \mathrm{ha}\right)$, frequência $(\%)$ e abundância (n/ha) bem distribuídas nas classes diamétricas, o que as torna uma fonte potencial de aproveitamento no futuro. Dados recentes publicados por EMBRAPA (2000) revelam que das quase 1000 espécies arbóreas das florestas da Amazônia Oriental, apenas 20 espécies madeireiras respondem por cerca de $80 \%$ do volume comercializado, o que demonstra que planos de manejo orientados sob aspectos puramente econômicos podem comprometer a base de recursos genéticos das espécies, devendo-se buscar alternativas que contribuam com a manutenção da viabilidade econômica do manejo florestal em regime de rendimento sustentado.

O método gráfico "Uplot-Dap" tem por finalidade auxiliar o pesquisador na análise exploratória das classes diamétricas, visando preparar os seus dados para estudos como distribuições diamétricas, relações hipsométricas e outros que necessitem de interpretação visual da dinâmica das classes diamétricas.

De acordo com Cunha et al. (2001), o "Uplot-Dap" pode ser utilizado em qualquer tipo de floresta, principalmente florestas naturais. $\mathrm{O}$ método é bastante flexível e permite que o pesquisador escolha a escala mais conveniente para expressar a dispersão dos diâmetros. $\mathrm{O}$ método utiliza-se do modo de dispersão $\mathrm{y}, \mathrm{x}$ para plotar os pontos e visualizar os dados num plano de observação dominados por pontos que se comportam como coordenadas vetoriais em sua respectiva classe diamétrica.

O objetivo da pesquisa foi detectar a existência e locação de um possível diâmetro máximo em função do equilíbrio biológico das espécies em uma floresta clímax, utilizando-se o método gráfico "Uplot-Dap".

\section{Material e Métodos}

\section{Base de Dados}

A área utilizada na presente 
pesquisa com destaque na (Fig. 1), tem formato retangular com $4 \mathrm{~km} \times 3,6$ $\mathrm{km}$, tem $1440 \mathrm{ha}$, compõe-se de 15 blocos contíguos de 96 ha de floresta primária, cada um medindo $800 \mathrm{~m}$ no sentido leste-oeste e $1200 \mathrm{~m}$ no sentido norte-sul e faz parte da Floresta Nacional do Tapajós (Flona Tapajós) que tem 600.000 ha e está situada entre os paralelos de $2^{\circ} 40^{\prime}$ a $4^{\circ} 10^{\prime}$ de latitude sul e os meridianos de $54^{\circ} 45^{\prime}$ a $55^{\circ} 30^{\prime}$ de longitude oeste de Greenwich, município de Belterra, Pará. O seu manejo está previsto para 2001, como parte do programa a ser executado pela Treviso Agropecuária Ltda., através de Convênio de Cooperação entre o Instituto Brasileiro do Meio Ambiente e dos Recursos Naturais Renováveis (IBAMA) e a International Tropical Timber Organization (ITTO), em andamento desde 1999.

De acordo com RADAMBRASIL (1976), predominam na área, os latossolos amarelos distróficos com diferentes texturas, profundos, ácidos, friáveis, onde ocorre a floresta densa. Como variação, tem-se também o latossolo amarelo distrófico plíntico de textura média e argilosa, os solos concrecionários lateríticos indiscriminados distróficos e areias quartzosas distróficas. Estes solos ocorrem em diversos tipos de relevo, variando desde plano a forte ondulado, sob vegetação de floresta densa e são originados a partir desedimentos do terciário.

A floresta ombrófila densa subdividida pelos quadrantes ecológicos (litologia, morfologia e clima), em termos de aspectos fisionômicos e florísticos, caracteriza a tipologia florestal dominante na região. Apresenta-se também na área uma físionomia florestal densa, com indivíduos emergentes, ocupando as áreas aplainadas dos platôs do XinguTapajós, caracterizada não só por apresentar alto volume, como também pela presença de espécies de alta cotação de preços nos mercados externo e interno (IBAMA/PNUD, 1997).

Os dados provêm do inventário florestal a $100 \%$ realizado pela Fundação de Pesquisas Florestais do Paraná (FUPEF) no ano de 1984 , mediante convênio firmado com o então Instituto Brasileiro de Desenvolvimento Florestal (IBDF). Foram utilizados os DAP $\geq 55 \mathrm{~cm}$ com casca de 28.320 árvores, provenientes de 15 blocos contíguos de floresta primária da Floresta Nacional do Tapajós (Fig. 1), medindo $800 \mathrm{~m} \mathrm{x}$ $1200 \mathrm{~m}$ (96ha), formando uma área retangular de $4 \mathrm{~km} \mathrm{x} 3,6 \mathrm{~km}$, totalizando $1440 \mathrm{ha}$, onde foram identificadas 210 espécies.

Testou-se o uso de valores aleatórios procedentes da distribuição uniforme $[0,1]$ e distribuição Beta $[\alpha, \beta]$ no cálculo da dispersão do diâmetro por permitirem que os valores da variável aleatória DAP se distribuam como eventos ou probabilidades mesmo em caso de distribuição truncada.

Para uma variável aleatória DAP 


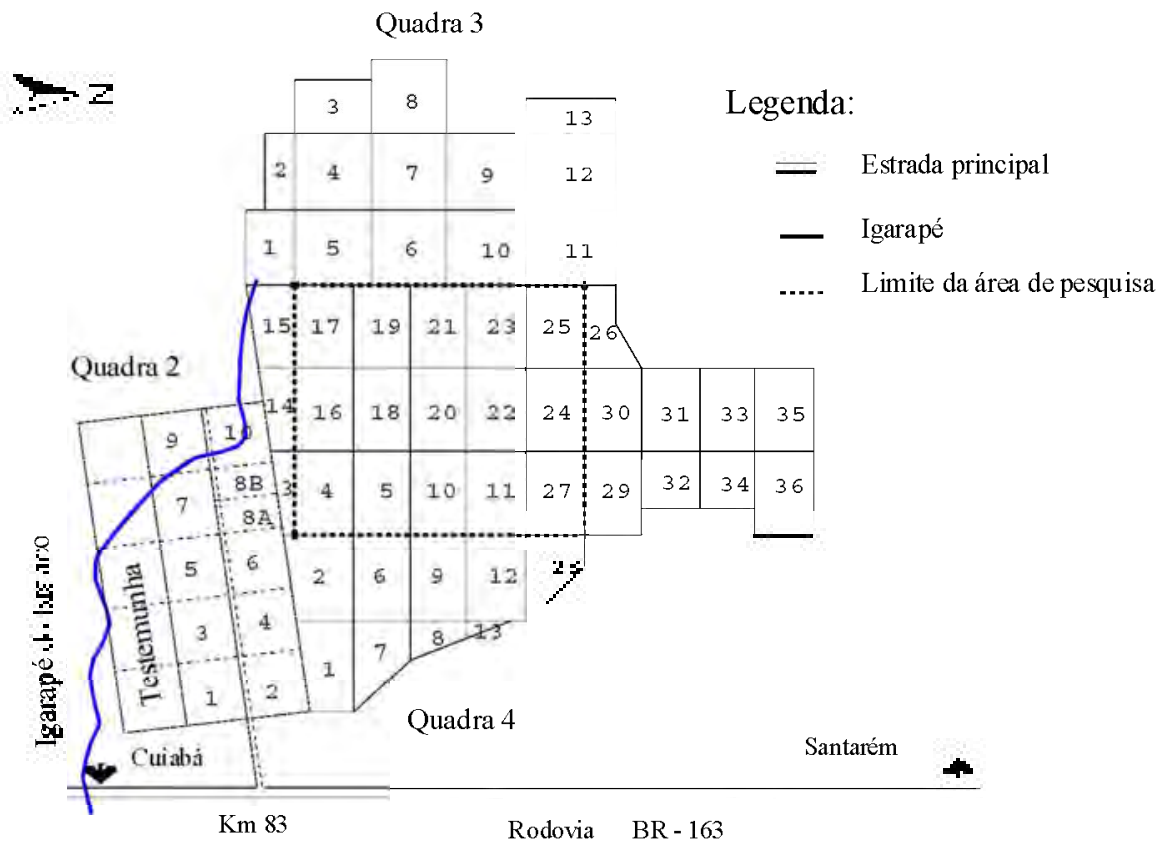

Figura 1. Localização e delimitação da área de pesquisa. Fonte: FUPEF (1984); IBAMA \& PNUD (1997).

$\mathrm{X}=$, distribuída uniformemente sobre $o$ intervalo $[\mathrm{a}, \mathrm{b}]$, onde $\mathrm{a}<\mathrm{b}$, tem-se que sua função densidade de probabilidade $(\mathrm{x}) \mathrm{f}$ é dada por:

$$
f(x)\left\{\begin{array}{lcc}
-\frac{1}{b-a} & \text { para } & a \leq x \leq b \\
-0 & \text { em outras circunstâncias }
\end{array}\right.
$$

Uma variável aleatória $\mathrm{X}=\mathrm{d}$ tem uma distribuição Beta com parâmetros $\alpha, \beta$ se sua função densidade de probabilidade $[f(d)]$ for dada por:

$$
f(d)-\frac{\Gamma(\alpha+\beta)}{\Gamma(\alpha) \Gamma(\beta)} d^{\alpha-1}(1-d)^{\beta-1} \quad \text { para } \quad 0<d<1
$$


em que

(d) $\mathrm{f}=$ função densidade de probabilidade $\alpha, \beta=$ parâmetros da função Beta $\Gamma(\cdot)=$ função gama,

$\Gamma(\alpha)=\int_{0}^{\infty} x^{\alpha-1} e^{-x} d x$ para $\alpha>0$ $\mathrm{d}=$ DAP transformado pela equação (3)

Obviamente que o emprego da função Beta expressa pela equação (2) no ajuste de distribuições diamétricas, exige que sejam feitas transformações nos dados que atendam ao campo de domínio da função. Como quase sempre acontece, os DAP medidos nos inventários começam a partir de valores como $10,20,30,35,45$ e $55 \mathrm{~cm}$, originando uma distribuição truncada. A principal vantagem no uso da função Beta está na facilidade de ajuste e no fato de que o ajuste para dados truncados não exige a recuperação dos parâmetros da distribuição uma vez que a transformação indicada na equação (3) envolvendo o DAP, executa uma translação de eixo que torna os limites dos dados compatíveis com o domínio da função:

\section{"Uplot-Dap}

Para o conjunto de DAP's de cada bloco, foram geradas duas funções visando à construção gráfica. A primeira, $\mathrm{f}(\mathrm{x})$, proporcionada pelo ajuste da distribuição Beta para os DAP observados. A segunda, $\mathrm{f}^{\prime}(\mathrm{x})$, resultante do produto da multiplicação de $f(x)$, função densidade de probabilidade da distribuição Beta, ou pelos números aleatórios da distribuição uniforme \{ a rand $[0,1]\}$ ou pelos números aleatórios da própria distribuição Beta $\{(a)$ rand $[\alpha, \beta]\}$, gerados usando-se os parâmetros estimados $\alpha, \beta$.

A seguir, plotou-se cada par de dados [DAP, $\left.f^{\prime}(x)\right]$, em modo de dispersão, o que resultou nos gráficos "Uplot-Dap", conforme pode ser observado nas Figuras 2, 3, 4 e 5 . Havendo necessidade de realçar a escala e melhorar a visualização dos pontos no gráfico, pode-se adicionar $\sqrt{\alpha}$ ao cálculo de $\mathrm{f}^{\prime}(\mathrm{x})$ resultando na seguinte expressão:

$\mathrm{f}^{\prime}(\mathrm{x})+\sqrt{\alpha}$.

O método deve seguir as

$$
\mathrm{d}=\left(\mathrm{DAP}+0,1-\mathrm{DAP}_{0}\right) /\left(\mathrm{DAP}_{+}+0,2-\mathrm{DAP}_{0}\right)
$$

em que

$\mathrm{d}=$ valor adimensional

DAP = diâmetro a altura do peito, $\mathrm{em} \mathrm{cm}$

$\mathrm{DAP}_{0}=$ menor DAP observado, em cm

$\mathrm{DAP}_{+}=$maior DAP observado, $\mathrm{em} \mathrm{cm}$

Princípios de Construção do seguintes fases e passos:

\section{Fase de Preparação dos Dados}

Passo 1. Utilizar a equação (3) para transformar a coleção de DAP $(\mathrm{cm})$, visando o ajuste da distribuição Beta. 
Passo 2. Ajustar a distribuição Beta para a obtenção dos parâmetros $\alpha$ e $\beta$.

Passo 3. Utilizar os parâmetros a e b para obter a geração de números aleatórios através da função Beta e para calcular a função densidade de probabilidade $\mathrm{f}(\mathrm{x})$. Em seguida, multiplicar $\mathrm{f}(\mathrm{x})$ por $@$, rand $[\alpha, \beta]$ ou por $@$, rand $[0,1]$, obtendo-se $\mathrm{f}^{\prime}(\mathrm{x})$. Portanto, observa-se que $\mathrm{f}^{\prime}(\mathrm{x})$ difere de $\mathrm{f}(\mathrm{x})$ apenas em termos do mecanismo aleatório responsável pela realização dos valores de $\mathrm{f}(\mathrm{x})$.

\section{Fase de Construção}

Passo 1. Plotar os pares [DAP, $\left.\mathrm{f}^{\prime}(\mathrm{x})\right]$ em modo de dispersão $\mathrm{xy}$, para obter o gráfico "Uplot-Dap".

Passo 2. Construir um histograma-base utilizando uma amplitude de referência com valores constantes de 5 ou $10 \mathrm{~cm}$. Recomendase usar a amplitude $5 \mathrm{~cm}$ para análises ao nível de espécies e $10 \mathrm{~cm}$ para a floresta como um todo.

Passo 3. Movimentar as classes de DAP (cm) de modo que o perfil observado no "Uplot-Dap" seja transcrito para o Histograma, conservando o máximo grau de semelhança com a forma explícita da distribuição dos pontos do "UplotDap". A movimentação das classes de DAP $(\mathrm{cm})$ significa fusão de classes adjacentes subsequentes com redimensionamento das amplitudes de classes ou até mesmo a manutenção da estrutura de classes desde que venham a ser consideradas uma característica típica da espécie ou da floresta.

Fase Final. Para que as alterações no histograma sejam consideradas satisfatórias é necessário que o perfil do histograma seja idêntico ao "Uplot-Dap" o que caracterizará o fim do processo, obtendo-se a distribuição das classes diamétricas construída com base em princípios gráficos.

\section{Resultados e Discussão}

Considerando que a aplicação do método para os 15 blocos apresentou resultados similares, decidiu-se escolher os blocos 10 com DAP $[55,155] \mathrm{cm}, 25 \mathrm{com}$ DAP $[55,195]$ $\mathrm{cm}, 18$ com DAP $[55,225] \mathrm{cm}$ e 24 com DAP $[55,265] \mathrm{cm}$ para demonstrar as técnicas de análise, visto que representam bem a variação total dos dados.

Analisando-se a Figura 2, observa-se que com o "Uplot-Dap" foi possível revelar uma faixa de pontos mais densa no entorno do DAP $=95 \mathrm{~cm}$ semelhante a um marcador de pontos enfileirados associados à uma nuvem de pontos agregados. Nota-se que a partir desse ponto, a quantidade de árvores por classe mantém a tendência decrescente, podendo-se visualizar classes com ausência ou presença rara de indivíduos.

Na Figura 3 é fácil perceber a formação nítida de um eixo de pontos na posição do $\mathrm{DAP}=95 \mathrm{~cm}$, sendo que a partir desse ponto as árvores que podem ser observadas estão distribuídas entre classes com vazios intermitentes e em alguns casos apresentam-se como pontos difusos.

Observa-se na Figura 4 a presença pendular de uma linha de 


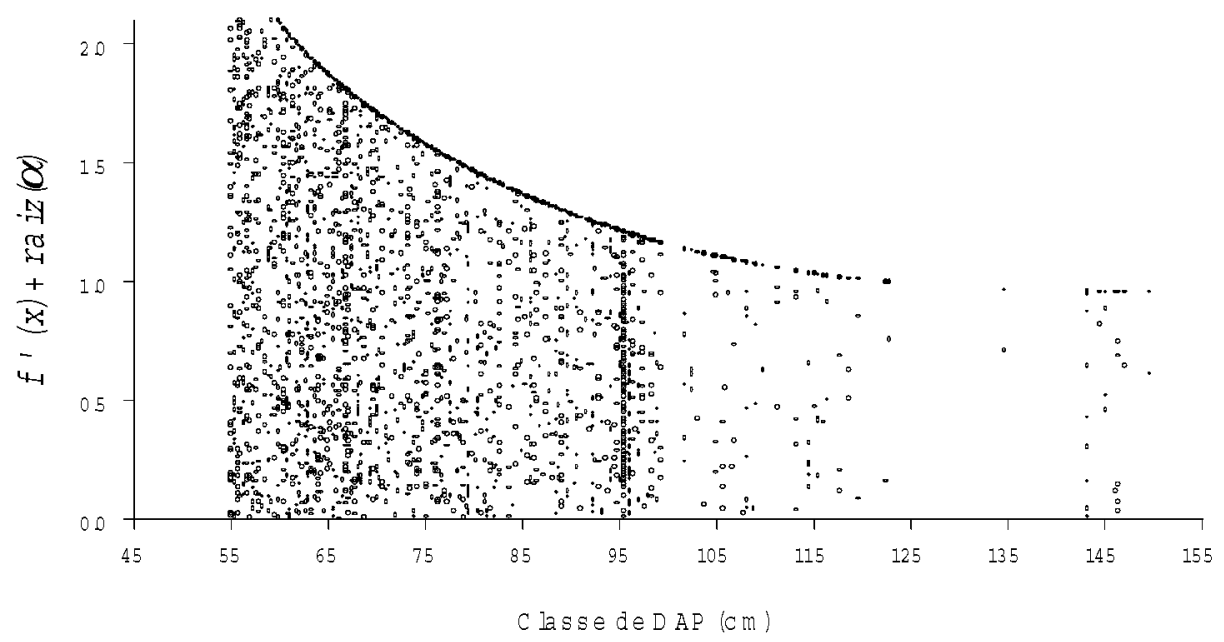

Figura 2. "Uplot-Dap" do bloco 10.

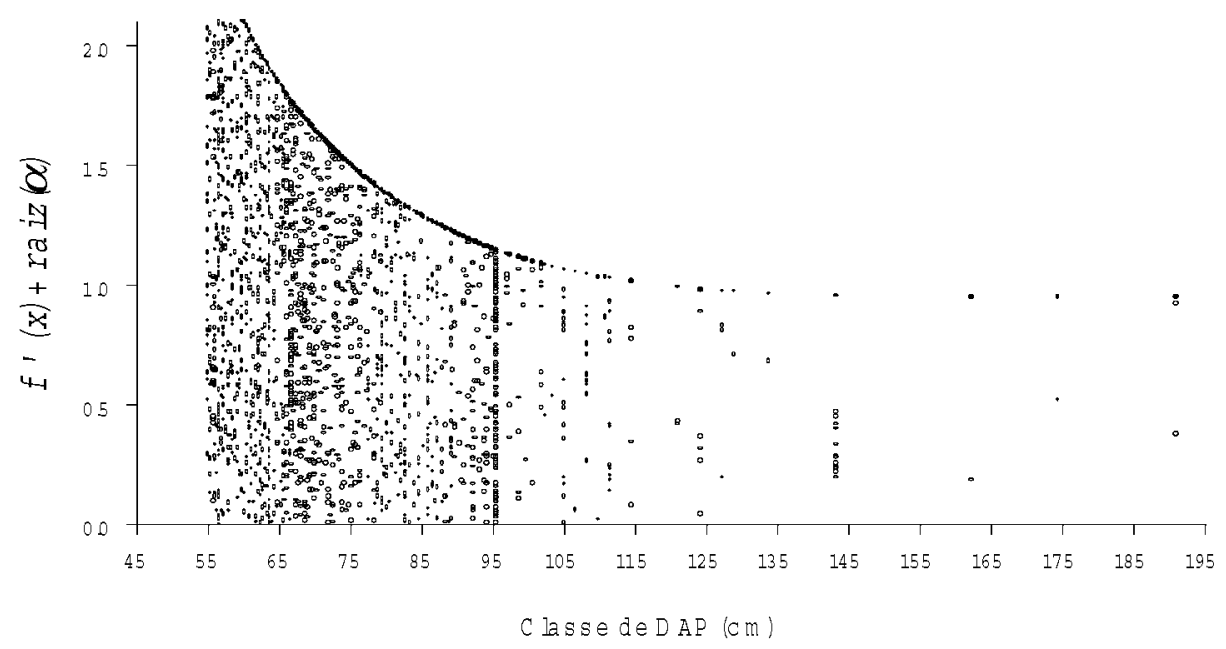

Figura 3. "Uplot-Dap" do bloco 25.

pontos na posição do $\mathrm{DAP}=95 \mathrm{~cm}$, marcando uma provável faixa de transição biológica das árvores clímax de várias espécies que conseguiram evoluir por seleção natural e competição dos espaços de crescimento disponíveis.

Na Figura 5, como nos casos anteriores, o efeito dos pontos gravitando no $\mathrm{DAP}=95$, não deixa dúvida quanto a possibilidade de tratar-se de um fenômeno biológico possível de ser identificado em função das relações entre os dados mostrado pelo "Uplot-Dap". Os valores do eixo da ordenada $\left(\mathrm{f}^{\prime}(\mathrm{x})+\sqrt{\alpha}\right)$ presentes nas Figuras $2,3,4$ e 5 refletem a variação 
probabilística@rand $(\cdot) \mathrm{x} f(\mathrm{x})$ entre 0 e 1 , por essa razão, ao adicionar a $\sqrt{\alpha}$, os valores dos limites superiores do eixo da ordenada são sempre maiores do que 1 .

Os resultados mostraram que o "Uplot-Dap" permite associar a variação diamétrica a fatores biológicos de crescimento e produção assintótica em florestas naturais clímax e que embora o DAP $=95$ tenha se repetido em todos os blocos, esse valor de referência pode variar de floresta para floresta.

Os resultados dos gráficos, mostraram também que o método

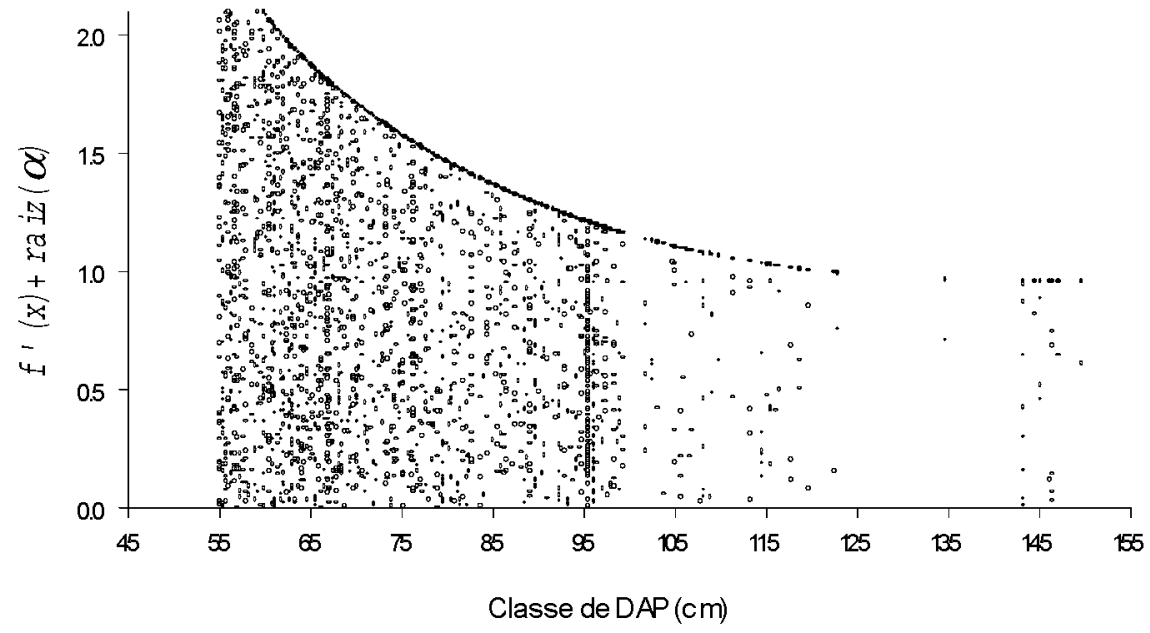

Figura 4. "Uplot-Dap" do bloco 18.

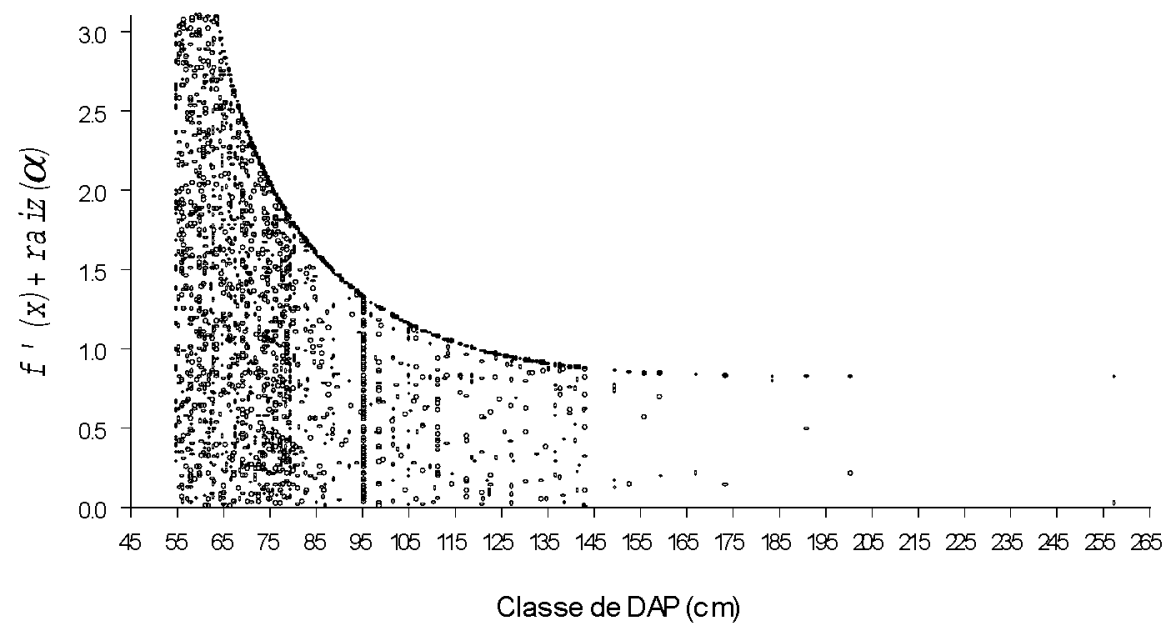

Figura 5. "uplot-dap" do bloco 24. 
permitiu identificar a existência de uma linha considerada como limite de transição do crescimento situada no entorno do DAP $=95 \mathrm{~cm}$. Em princípio trata-se de um ponto de convergência estrutural que registra a evolução de várias espécies ao longo do processo de desenvolvimento da floresta. Esse ponto poderá variar de floresta para floresta e sua nitidez depende do tipo de floresta e suas condições estruturais. Somente em florestas primárias em estágio clímax é possível identificar esse aspecto.

Mesmo mantendo-se uma distribuição regular de árvores a partir da classe de DAP $=95 \mathrm{~cm}$, a viabilidade em usar esse diâmetro como valor de referência, baseia-se nos seguintes argumentos: i) apesar desse limite apresentar-se de forma difusa, uniforme ou irregular, sua detecção permitirá a priori se reorientar as bases do manejo florestal, na medida em que a maioria das florestas manejada da Amazônia ainda estão atravessando o primeiro ciclo de corte, sendo necessário, portanto, precaução para que não se arbitre uma intensidade de exploração acima da capacidade de recuperação da floresta; ii) os resultados desta pesquisa mostraram que, em média, o total de árvores que ultrapassaram o $\mathrm{DAP}=95$ $\mathrm{cm}$ foi da ordem de $11,09 \%$ para uma área comercial de 1440 ha com 15 blocos de 96 ha. Isto significa que o total das árvores com diâmetro variando de $55 \mathrm{~cm}$ a $95 \mathrm{~cm}$, corresponde a $90 \%$ da população de indivíduos com dimensões comerciais. Além disso, árvores com grandes diâmetros causam maior impacto ambiental na exploração formando grandes clareiras, causando problemas operacionais no arraste e em alguns casos baixo aproveitamento comercial.

Embora ainda não seja possível fazer a seleção mais adequada das árvores porta-sementes com base em critérios mais consistentes como sistema reprodutivo e estado fitossanitário, as árvores de grande porte podem assumir o papel de portasementes e podem ajudar de várias formas na recuperação da floresta, pois são fontes de diversidade biogenética de elevado valor estratégico em termos de regulação da produção florestal.

\section{Conclusão}

A pesquisa produziu a seguinte conclusão:

Existem evidências de que o $\mathrm{DAP}=95 \mathrm{~cm}$ para esse tipo de floresta, poderá ser estabelecido como um possível indicador do diâmetro máximo de explorabilidade.

\section{Recomendação}

Recomenda-se manter o diâmetro máximo de referência de corte com valor fixo de $95 \mathrm{~cm}$ na primeira intervenção, como medida de controle na recuperação da floresta em questão.

\section{Agradecimentos}

O autor agradece a colaboração do Prof. Dr. Jayme Cardoso, especialista em Neográfica, por suas sugestões valiosas e comentários, o que muito contribuiu para melhorar e enriquecer a qualidade final do 
presente artigo.

\section{Bibliografia citada}

Cunha, U. S. ; Machado, S. A. ; Figueiredo Filho, A.; Hosokawa, R. T. Novo método "UPLOT-DAP" para representação gráfica de distribuições diamétricas. Curitiba-PR, Floresta. 2001 (Submetido).

Eddowes, P. J. Technical aspects of marketing unfamiliar species. Trabajo presentado en la $11^{\text {a }}$ Conferencia Forestal del Common wealth, Trinidad y Tabago. 1980.

Embrapa. Relatório do workshop de implementação do projeto Dendrogene: conservação genética em florestas manejadas na Amazônia. Belém, 2000, $88 \mathrm{p}$.

Fundação de Pesquisas Florestais do ParanáFUPEF. Inventário comercial da quadra de exploração $n^{\circ} 4$ da floresta nacional do tapajós. Curitiba, 1984. 429p.

IBAMA/PNUD. Plano de manejo florestal da floresta nacional do Tapajós, Pará, Brasil. Belém, 1997. 109p.

RADAMBRASIL. Projeto RADAMBRASIL. Departamento Nacional da Produção Mineral, Folha SA 21-Santarém, Rio de Janeiro, 1976 (Levantamento de Recursos Naturais, 10).

Fundação de Pesquisas Florestais do ParanáFUPEF. Inventário comercial da quadra de exploração $n^{\circ} 4$ da Floresta Nacional do Tapajós. Curitiba, 1984. 429p.

Aceito para publicação em 04/07/2002 
